\title{
MICRONUTRIENT CATIONS DISTRIBUTION IN THE SOIL PROFILE OF ORANGE (Citrus reticulata) ORCHARD OF TAMENGLONG DISTRICT, MANIPUR (INDIA)
}

\author{
Herojit Singh Athokpam ${ }^{1}$, Konsam Vikramjeet ${ }^{1}$, Nandini Chongtham ${ }^{2}$, K. Nandini Devi ${ }^{1}$, \\ Naorem Brajendra Singh ${ }^{1}$, N. Gopimohan Singh ${ }^{1}$, P.T. Sharma ${ }^{1}$ and Punabati Heisnam ${ }^{3}$
}

${ }^{1}$ College of Agriculture, Central Agricultural University, Imphal - 795004, India

${ }^{2}$ kvk, Central Agricultural University, Imphal - 795004, India

${ }^{3}$ College of Agriculture, Central Agricultural University, Pasighat - 795004, India

Received - October 31, 2017; Revision - December 11, 2017; Accepted - January 06, 2018

Available Online - February 20, 2018

DOI: http://dx.doi.org/10.18006/2018.6(1).108.115

\section{KEYWORDS \\ DTPA \\ Micronutrient \\ Cations \\ Orange orchard \\ Profile}

\begin{abstract}
Vertical distribution of DTPA-extractable micronutrient cations ( $\mathrm{Zn}, \mathrm{Cu}, \mathrm{Fe}$ and $\mathrm{Mn}$ ) and their relationship with various soil properties were studied in sixteen profiles of orange orchard of Tamenglong district of Manipur. The DTPA-extractable $\mathrm{Zn}, \mathrm{Cu}, \mathrm{Fe}$ and $\mathrm{Mn}$ content were greater in the surface soils than the sub-surface horizons. In most of the profiles the value of $\mathrm{Zn}, \mathrm{Cu}, \mathrm{Fe}$ and $\mathrm{Mn}$ ranged from 0.20 to 1.75 , trace to $0.80,5.80$ to 42.10 and 0.35 to $39.55 \mathrm{mg} \mathrm{kg}^{-1}$, respectively. The content of DTPA-extractable $\mathrm{Zn}, \mathrm{Cu}, \mathrm{Fe}$ and $\mathrm{Mn}$ were higher surface and gradually decreased with the depth. DTPA-extractable $\mathrm{Zn}$ was found deficient in 73 per cent, marginal in 16 per cent and sufficient in 1.50 per cent in the soil samples while $\mathrm{Cu}, \mathrm{Fe}$ and $\mathrm{Mn}$ were sufficient in all soils except one profile in $\mathrm{Cu}$ and $\mathrm{Mn}$. Multiple regression co-efficient analysis showed that the extractable $\mathrm{Zn}, \mathrm{Cu}, \mathrm{Fe}$ and $\mathrm{Mn}$ content were influenced by silt, $\mathrm{EC}, \mathrm{Al}_{2} \mathrm{O}_{3}$ and $\mathrm{Mg}$ to the level of $0.77,0.80,0.73$ and 0.66 , respectively. However, these micronutrient cations were significantly contributed only by silt and EC.
\end{abstract}

* Corresponding author

E-mail: hathokpam@yahoo.in (Herojit Singh Athokpam)

Peer review under responsibility of Journal of Experimental Biology and Agricultural Sciences.

Production and Hosting by Horizon Publisher India [HPI] (http://www.horizonpublisherindia.in/).

All rights reserved.
All the article published by Journal of Experimental Biology and Agricultural Sciences is licensed under a Creative Commons Attribution-NonCommercial 4.0 International License Based on a work at www.jebas.org.

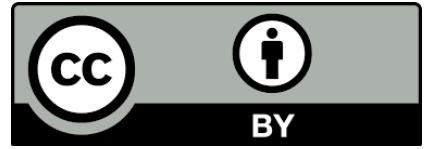




\section{Introduction}

Role of micronutrients in balanced plant nutrition is well established. Micronutrients are very important for maintaining soil health and also in increasing productivity of crops (Rattan et al., 2009). Application of $\mathrm{Mg}+\mathrm{Cu}+\mathrm{Zn}$ gave the highest fruit yield of orange but did not affect fruit quality (Ram \& Bose, 2000). Sweet orange fruit yield was increased with foliar application of $0.4 \mathrm{~kg} \mathrm{Zn} \mathrm{ha}^{-1}$ and $0.2 \mathrm{~kg} \mathrm{Mn} \mathrm{ha}^{-1}$ in the presence of $1.56 \mathrm{~kg} \mathrm{~N} \mathrm{ha}^{-1}$ (Tariq et al., 2007). Foliar spray of $\mathrm{CuSO}_{4}(0.4 \%)$ at pea stage and gravel stage increased the fruit retention of Nagpur mandarin was reported by Soni et al. (2017). Flourishes in the growth and yield attributes of Mandarin orange was noticed with foliar spray of micronutrients (Kumar et al., 2017). Foliar application of micronutrients gave the higher Kinnow mandarin fruit yield than the soil treatment under semi-arid zone (Vijaya et al., 2017). However, exploitive nature of modern agriculture involving use of high analysis NPK fertilizers coupled with limited use of organic manure and less recycling of crop residues are important factors contributing towards accelerated exhaustion of micronutrients from the soil (Sharma \& Choudhary, 2007). Continuous negligence of micronutrient application and avoidance of organic manures are the major causes of deficiency of these micronutrients (Srivastava et al., 2017). Moreover, citrus is deep rooted plant, micronutrients application at the soil surface may be of a little value. Therefore, a major constraint for productivity and sustainability of the Indian soils were due to the deficiency of micronutrients in the surface soil as well as sub-surface soil. The availability of micronutrients to plants is also influenced by the distribution within the soil profile (Singh \& Dhankar, 1989). The knowledge of pedogenic distribution of micronutrients is important as many plant roots penetrate to the sub-surface layers and thus, draw a part of the nutrient requirement from the subsurface horizon of the soils. The distribution of micronutrient cations of orange orchards of Tamenglong district of Manipur was not yet studied. Therefore, the present work has been undertaken to assess the distribution of micronutrient cations of the orange orchards and to find out the relationship between the soil properties and micronutrients.

\section{Materials and Methods}

The studied area lies between $24^{0} 45^{\prime} \mathrm{N}$ to $24^{0} 59^{\prime} \mathrm{N}$ latitudes and $93^{0} 26^{\prime}$ longitudes and located in South-western part of Manipur, India with an elevation of 900-1200 m MSL. Sixteen typical soil profiles from different orchards of Tamenglong District, Manipur were exposed and soil samples were collected depth-wise i.e. 020, 20-40, 40-60 and 60-80 cm. in the clean polythene bags. All the composite soil samples were air-dried in the shade, ground and passed through $2 \mathrm{~mm}$ sieve for chemical analysis. Typical soil profiles were exposed by collecting depth wise i.e. 0-20, 20-40, $40-60$ and $60-80 \mathrm{~cm}$ soil samples. These were processed and analyzed for various physicochemical properties like sand, silt, clay content, $\mathrm{pH}$, EC (1:2.5 soil: water), organic carbon, CEC, available $\mathrm{N}, \mathrm{P}$ and $\mathrm{K}, \mathrm{Al}_{2} \mathrm{O}_{3}$ and $\mathrm{Fe}_{2} \mathrm{O}_{3}$ using standard laboratory procedures outline by Jackson (1973), Borah et al. (1987) and Chopra \& Kanwar (1976).

The DTPA-extractable $\mathrm{Zn}, \mathrm{Cu}, \mathrm{Fe}$ and $\mathrm{Mn}$ in the soil samples were extracted with a solution of $0.005 \mathrm{M}$ DTPA, $0.01 \mathrm{M} \mathrm{CaCl}_{2}$ and $0.1 \mathrm{M}$ tri-ethanolamine adjusted to $\mathrm{pH} 7.3$ as outlined by Lindsay \& Norvell (1978). This micronutrient cations concentration in the soils was analyzed by using AAS. Multiple regression equations were computed between DTPA-extractable micronutrients and soil properties by adopting statistical procedures (Panse \& Sukhatme, 1961).

\section{Results and Discussion}

The relevant soil characteristics of the representative soil profiles are describe in Table 1. No definite pattern was found in the distribution of sand, silt, and clay content in the profile i.e. 11.8 to 45.2, 5.0 to 32.9 and 37.3 to 62.3 per cent, respectively. The EC of the soils varied from 0.011 to $0.256 \mathrm{dSm}^{-1}$ and soil organic carbon ranged from 4.2 to $18.6 \mathrm{~g} \mathrm{~kg}^{-1}$. Organic carbon in surface soil layers was more than the sub-surface layers. CEC ranged from 9.6 to $24.0\left[\mathrm{cmol}\left(\mathrm{p}^{+}\right)\right] \mathrm{kg}^{-1}$ soil. Free oxides of iron and aluminum varied from 0.2 to 0.6 and 5.7 to 13.5 per cent, respectively. The exchangeable $\mathrm{Ca}$ and $\mathrm{Mg}$ content in the soils were 0.39 to 7.85 and 0.13 to $5.00\left[\mathrm{cmol}\left(\mathrm{p}^{+}\right)\right] \mathrm{kg}^{-1}$ soil, respectively, both bases decreased with increased in depth in all the soil profiles. The available N, P and K content in the soils were 125.0 to $313.0,2.2$ to 22.4 and 39.2 to $269.0 \mathrm{~kg} \mathrm{ha}^{-1}$, respectively. These nutrients content decreased with increased the depth in the profile.

\subsection{Zinc (Zn)}

DTPA-extractable $\mathrm{Zn}$ in the studied soil profiles varied from trace to $1.75 \mathrm{mg} \mathrm{kg}^{-1}$ in the orange growing soils of Tamenglong district of Manipur. Sen et al. (1997) reported the available $\mathrm{Zn}$ content varies from 0.2 to $1.4 \mathrm{mg} \mathrm{kg}^{-1}$ and decreased down the profile (Khanday et al., 2017). Similar report was also reported by Athokpam et al. (2016) in the citrus orchard of Ukhrul district, Manipur. Considering $0.6 \mathrm{mg} \mathrm{kg}^{-1}$ as the critical limit of available $\mathrm{Zn}$ as suggested by Takkar \& Mann (1975), 73.4, 25.0 and 1.6 percent of the studied soil samples fell in deficient, marginal and sufficient categories, respectively. DTPA-extractable Zn showed significant regression (Table 3 ) with EC $(0.868 *)$ and $\mathrm{Fe}(0.024 *)$. 
Table 1 Physico-chemical properties of the soil profiles

\begin{tabular}{|c|c|c|c|c|c|c|c|c|c|c|c|c|c|c|c|}
\hline $\begin{array}{l}\text { Depth } \\
(\mathrm{cm})\end{array}$ & $\begin{array}{c}\text { Sand } \\
(\%)\end{array}$ & $\begin{array}{l}\text { Silt } \\
(\%)\end{array}$ & $\begin{array}{l}\text { Clay } \\
(\%)\end{array}$ & Texture & $\mathrm{pH}$ & $\begin{array}{c}\mathrm{EC} \\
(\mathrm{dS} \\
\left.\mathrm{m}^{-1}\right)\end{array}$ & $\underset{\left(\mathrm{gkg}^{-1}\right)}{\mathrm{OC}}$ & $\begin{array}{c}\mathrm{CEC} \\
{[\mathrm{cmol}} \\
\left(\mathrm{p}^{+}\right) \\
\left.\mathrm{kg}^{-1}\right]\end{array}$ & $\begin{array}{c}\mathrm{Fe}_{2} \mathrm{O}_{3} \\
(\%)\end{array}$ & $\begin{array}{c}\mathrm{Al}_{2} \mathrm{O}_{3} \\
(\%)\end{array}$ & $\begin{array}{c}\mathrm{Ca} \\
{[\mathrm{cmol}(} \\
\left.\mathrm{p}^{+}\right) \\
\left.\mathrm{kg}^{-1}\right]\end{array}$ & $\begin{array}{c}\mathrm{Mg} \\
{[\mathrm{cmol}} \\
\left(\mathrm{p}^{+}\right) \\
\left.\mathrm{kg}^{-1}\right]\end{array}$ & $\begin{array}{c}\mathrm{N} \\
(\mathrm{kg} \\
\left.\mathrm{ha}^{-1}\right)\end{array}$ & $\begin{array}{c}\mathrm{P} \\
(\mathrm{kg} \\
\left.\mathrm{ha}^{-1}\right)\end{array}$ & $\begin{array}{c}\mathrm{K} \\
(\mathrm{kg} \\
\left.\mathrm{ha}^{-1}\right)\end{array}$ \\
\hline \multicolumn{16}{|c|}{ Profile 1(Longmai Noney II) } \\
\hline $0-20$ & 39.3 & 22.5 & 38.2 & $\mathrm{CL}$ & 4.70 & 0.084 & 1.41 & 15.2 & 0.56 & 6.7 & 0.93 & 2.70 & 250.88 & 15.68 & 95.2 \\
\hline $20-40$ & 36.8 & 25.0 & 38.2 & $\mathrm{CL}$ & 4.80 & 0.032 & 0.66 & 14.0 & 0.48 & 6.2 & 0.73 & 1.92 & 313.60 & 13.44 & 50.4 \\
\hline $40-60$ & 36.8 & 22.5 & 40.7 & C4.9 & 4.90 & 0.015 & 0.51 & 13.6 & 0.32 & 6.0 & 1.00 & 1.93 & 188.16 & 13.44 & 39.2 \\
\hline $60-80$ & 39.3 & 17.5 & 43.2 & $\mathrm{C}$ & 5.00 & 0.015 & 0.42 & 14.8 & 0.32 & 6.1 & 0.80 & 2.13 & 188.16 & 11.20 & 44.8 \\
\hline \multicolumn{16}{|c|}{ Profile 2 (Longmai Noney II) } \\
\hline $0-20$ & 26.7 & 24.7 & 48.6 & $\mathrm{C}$ & 4.84 & 0.098 & 1.86 & 16.0 & 0.56 & 5.8 & 1.60 & 1.26 & 313.60 & 11.20 & 123.2 \\
\hline $20-40$ & 23.5 & 32.9 & 43.6 & $\mathrm{C}$ & 4.85 & 0.033 & 1.26 & 14.0 & 0.48 & 7.2 & 1.00 & 0.46 & 188.16 & 6.72 & 78.4 \\
\hline $40-60$ & 36.0 & 20.4 & 43.6 & $\mathrm{C}$ & 4.82 & 0.022 & 1.20 & 15.8 & 0.44 & 7.1 & 0.86 & 0.86 & 188.16 & 4.48 & 72.8 \\
\hline $60-80$ & 33.5 & 22.5 & 44.0 & $\mathrm{C}$ & 4.84 & 0.019 & 1.20 & 13.8 & 0.52 & 6.3 & 0.93 & 0.80 & 125.44 & 2.24 & 72.8 \\
\hline \multicolumn{16}{|c|}{ Profile 3 (Longmai Noney I) } \\
\hline $0-20$ & 24.3 & 25.0 & 50.7 & $\mathrm{C}$ & 4.71 & 0.103 & 1.86 & 17.0 & 0.36 & 11.9 & 1.73 & 1.70 & 313.60 & 13.44 & 224.0 \\
\hline $20-40$ & 26.4 & 15.9 & 57.7 & $\mathrm{C}$ & 4.82 & 0.043 & 1.32 & 17.2 & 0.32 & 13.5 & 0.66 & 1.33 & 313.60 & 8.96 & 128.0 \\
\hline $40-60$ & 23.5 & 25.6 & 50.9 & $\mathrm{C}$ & 4.85 & 0.023 & 1.02 & 16.8 & 0.36 & 13.3 & 0.73 & 0.73 & 125.44 & 4.48 & 95.2 \\
\hline $60-80$ & 23.5 & 25.6 & 50.9 & $\mathrm{C}$ & 4.90 & 0.020 & 0.78 & 15.0 & 0.40 & 8.6 & 0.66 & 0.72 & 188.16 & 6.72 & 106.4 \\
\hline \multicolumn{16}{|c|}{ Profile 4 (Longmai Noney I) } \\
\hline $0-20$ & 19.3 & 27.5 & 53.2 & $\mathrm{C}$ & 4.60 & 0.068 & 1.71 & 23.6 & 0.52 & 6.6 & 1.33 & 3.33 & 313.60 & 8.96 & 184.8 \\
\hline $20-40$ & 11.8 & 30.0 & 58.2 & $\mathrm{C}$ & 4.63 & 0.033 & 1.26 & 19.2 & 0.44 & 8.6 & 0.86 & 1.00 & 250.88 & 4.48 & 140.0 \\
\hline $40-60$ & 15.2 & 27.5 & 57.3 & $\mathrm{C}$ & 4.64 & 0.020 & 0.90 & 18.6 & 0.32 & 8.4 & 0.80 & 0.26 & 188.16 & 4.48 & 112.0 \\
\hline $60-80$ & 12.7 & 25.0 & 62.3 & $\mathrm{C}$ & 4.61 & 0.050 & 0.66 & 17.6 & 0.36 & 9.0 & 0.66 & 0.76 & 125.44 & 6.72 & 117.6 \\
\hline \multicolumn{16}{|c|}{ Profile 5 (Tupul I) } \\
\hline $0-20$ & 35.2 & 22.5 & 42.3 & $\mathrm{C}$ & 5.20 & 0.210 & 2.16 & 24.0 & 0.56 & 6.7 & 7.85 & 3.30 & 250.88 & 22.40 & 240.8 \\
\hline $20-40$ & 42.7 & 17.5 & 39.8 & $\mathrm{SC}$ & 5.30 & 0.216 & 1.50 & 20.4 & 0.44 & 9.2 & 6.33 & 3.93 & 250.88 & 13.40 & 184.8 \\
\hline $40-60$ & 37.7 & 20.0 & 42.3 & $\mathrm{C}$ & 5.20 & 0.214 & 1.41 & 19.2 & 0.48 & 9.1 & 6.10 & 3.30 & 188.16 & 13.40 & 173.6 \\
\hline $60-80$ & 45.2 & 17.5 & 37.3 & SCL & 4.82 & 0.050 & 0.96 & 13.6 & 0.24 & 9.4 & 4.20 & 3.30 & 188.16 & 8.96 & 128.0 \\
\hline \multicolumn{16}{|c|}{ Profile 6(Tupul II) } \\
\hline $0-20$ & 21.4 & 30.0 & 48.6 & $\mathrm{C}$ & 4.80 & 0.130 & 2.01 & 20.0 & 0.44 & 7.9 & 3.50 & 3.40 & 250.88 & 17.92 & 102.4 \\
\hline $20-40$ & 23.5 & 27.9 & 48.6 & $\mathrm{C}$ & 4.84 & 0.079 & 1.53 & 19.4 & 0.48 & 9.0 & 1.60 & 2.90 & 313.60 & 13.44 & 112.0 \\
\hline $40-60$ & 30.5 & 25.9 & 43.6 & $\mathrm{C}$ & 4.85 & 0.049 & 1.02 & 14.0 & 0.48 & 9.1 & 1.90 & 1.50 & 250.88 & 13.44 & 78.4 \\
\hline $60-80$ & 27.3 & 26.2 & 46.5 & $\mathrm{C}$ & 4.82 & 0.050 & 0.96 & 13.6 & 0.40 & 7.7 & 1.00 & 1.60 & 250.88 & 8.96 & 89.6 \\
\hline \multicolumn{16}{|c|}{ Profile 7 (Nungtek) } \\
\hline $0-20$ & 29.4 & 22.0 & 48.6 & $\mathrm{C}$ & 4.60 & 0.256 & 1.56 & 16.4 & 0.48 & 5.7 & 5.80 & 2.50 & 313.60 & 15.68 & 257.6 \\
\hline $20-40$ & 32.0 & 22.0 & 46.0 & $\mathrm{C}$ & 4.90 & 0.088 & 0.87 & 14.4 & 0.36 & 8.4 & 3.00 & 1.95 & 250.88 & 13.44 & 140.0 \\
\hline $40-60$ & 32.1 & 26.8 & 41.1 & $\mathrm{C}$ & 4.80 & 0.045 & 0.63 & 12.4 & 0.28 & 7.6 & 1.90 & 0.99 & 250.88 & 8.96 & 67.2 \\
\hline $60-80$ & 40.5 & 19.3 & 40.2 & C & 4.70 & 0.040 & 0.57 & 12.0 & 0.20 & 6.7 & 1.50 & 1.26 & 188.16 & 11.20 & 78.4 \\
\hline
\end{tabular}




\begin{tabular}{|c|c|c|c|c|c|c|c|c|c|c|c|c|c|c|c|}
\hline $\begin{array}{l}\text { Depth } \\
(\mathrm{cm})\end{array}$ & $\begin{array}{l}\text { Sand } \\
(\%)\end{array}$ & $\begin{array}{l}\text { Silt } \\
(\%)\end{array}$ & $\begin{array}{l}\text { Clay } \\
(\%)\end{array}$ & Texture & $\mathrm{pH}$ & $\begin{array}{c}\mathrm{EC} \\
(\mathrm{dS} \\
\left.\mathrm{m}^{-1}\right)\end{array}$ & $\underset{\left(\mathrm{gkg}^{-1}\right)}{\mathrm{OC}}$ & $\begin{array}{c}\text { CEC } \\
{[\mathrm{cmol}} \\
\left(\mathrm{p}^{+}\right) \\
\left.\mathrm{kg}^{-1}\right]\end{array}$ & $\begin{array}{c}\mathrm{Fe}_{2} \mathrm{O}_{3} \\
(\%)\end{array}$ & $\begin{array}{c}\mathrm{Al}_{2} \mathrm{O}_{3} \\
(\%)\end{array}$ & $\begin{array}{c}\mathrm{Ca} \\
{[\mathrm{cmol}} \\
\left(\mathrm{p}^{+}\right) \\
\left.\mathrm{kg}^{-1}\right]\end{array}$ & $\begin{array}{c}\mathrm{Mg} \\
{[\mathrm{cmol}} \\
\left(\mathrm{p}^{+}\right) \\
\left.\mathrm{kg}^{-1}\right]\end{array}$ & $\begin{array}{c}\mathrm{N} \\
(\mathrm{kg} \\
\left.\mathrm{ha}^{-1}\right)\end{array}$ & $\begin{array}{c}\mathrm{P} \\
(\mathrm{kg} \\
\left.\mathrm{h}^{-1}\right)\end{array}$ & $\begin{array}{c}\mathrm{K} \\
(\mathrm{kg} \\
\left.\mathrm{ha}^{-1}\right)\end{array}$ \\
\hline \multicolumn{16}{|c|}{ Profile 8 (Thangal) } \\
\hline $0-20$ & 25.5 & 18.4 & 56.1 & $\mathrm{C}$ & 4.80 & 0.252 & 2.16 & 22.4 & 0.60 & 6.6 & 5.80 & 4.80 & 313.60 & 22.4 & 268.8 \\
\hline $20-40$ & 28.0 & 25.9 & 46.1 & $\mathrm{C}$ & 4.60 & 0.155 & 1.32 & 20.0 & 0.48 & 5.9 & 4.32 & 4.20 & 313.60 & 17.92 & 257.6 \\
\hline $40-60$ & 40.5 & 15.9 & 43.6 & $\mathrm{C}$ & 4.60 & 0.072 & 0.81 & 16.4 & 0.44 & 6.6 & 2.93 & 3.40 & 250.88 & 13.44 & 190.4 \\
\hline $60-80$ & 38.9 & 15.9 & 45.2 & $\mathrm{C}$ & 4.70 & 0.040 & 0.60 & 14.8 & 0.32 & 7.8 & 2.33 & 3.26 & 188.16 & 15.68 & 117.6 \\
\hline \multicolumn{16}{|c|}{ Profile 9 (Awang Khul) } \\
\hline $0-20$ & 33.9 & 17.5 & 48.6 & $\mathrm{C}$ & 4.20 & 0.104 & 2.16 & 14.8 & 0.32 & 7.2 & 0.93 & 0.79 & 250.88 & 20.16 & 145.6 \\
\hline $20-40$ & 32.5 & 16.0 & 51.5 & $\mathrm{C}$ & 4.40 & 0.070 & 1.20 & 13.0 & 0.48 & 10.5 & 0.73 & 0.79 & 250.88 & 17.92 & 84.0 \\
\hline $40-60$ & 33.5 & 5.0 & 61.5 & $\mathrm{C}$ & 4.60 & 0.021 & 0.66 & 12.8 & 0.36 & 9.3 & 0.53 & 0.26 & 188.16 & 11.20 & 72.8 \\
\hline $60-80$ & 29.0 & 20.0 & 51.0 & $\mathrm{C}$ & 4.60 & 0.043 & 0.45 & 9.6 & 0.36 & 9.6 & 0.39 & 0.39 & 188.16 & 8.96 & 84.0 \\
\hline \multicolumn{16}{|c|}{ Profile 10 (Rengpang) } \\
\hline $0-20$ & 16.0 & 28.8 & 55.2 & $\mathrm{C}$ & 4.30 & 0.065 & 1.71 & 16.4 & 0.44 & 8.2 & 1.19 & 0.66 & 250.88 & 20.16 & 246.4 \\
\hline $20-40$ & 25.3 & 15.2 & 59.5 & $\mathrm{C}$ & 4.40 & 0.052 & 0.96 & 14.4 & 0.56 & 9.4 & 1.19 & 0.46 & 250.88 & 17.92 & 123.2 \\
\hline $40-60$ & 25.3 & 16.8 & 57.9 & $\mathrm{C}$ & 4.50 & 0.012 & 0.54 & 13.8 & 0.48 & 8.7 & 0.79 & 0.26 & 188.16 & 17.92 & 100.8 \\
\hline $60-80$ & 18.4 & 26.3 & 55.3 & $\mathrm{C}$ & 4.60 & 0.011 & 0.51 & 13.2 & 0.40 & 9.0 & 0.73 & 0.26 & 125.44 & 15.68 & 95.2 \\
\hline \multicolumn{16}{|c|}{ Profile 11 (Longmai I) } \\
\hline $0-20$ & 32.7 & 22.5 & 44.8 & $\mathrm{C}$ & 4.60 & 0.084 & 1.08 & 14.4 & 0.48 & 8.7 & 2.30 & 2.90 & 313.60 & 17.92 & 95.2 \\
\hline $20-40$ & 27.7 & 22.5 & 49.8 & $\mathrm{C}$ & 4.70 & 0.042 & 0.78 & 14.0 & 0.36 & 11.1 & 2.10 & 2.90 & 250.88 & 17.92 & 67.2 \\
\hline $40-60$ & 25.2 & 25.0 & 49.8 & $\mathrm{C}$ & 4.80 & 0.045 & 0.75 & 12.8 & 0.32 & 10.2 & 1.70 & 3.19 & 188.16 & 15.68 & 67.2 \\
\hline $60-80$ & 30.2 & 27.5 & 42.3 & $\mathrm{C}$ & 4.80 & 0.023 & 0.81 & 12.0 & 0.32 & 9.9 & 1.30 & 2.53 & 188.16 & 13.44 & 72.8 \\
\hline \multicolumn{16}{|c|}{ Profile 12 (Longmai II) } \\
\hline $0-20$ & 29.1 & 30.9 & 40.0 & $\mathrm{C}$ & 4.70 & 0.060 & 1.38 & 16.0 & 0.52 & 6.4 & 2.70 & 5.00 & 188.16 & 8.96 & 162.4 \\
\hline $20-40$ & 37.5 & 20.0 & 42.5 & $\mathrm{C}$ & 4.80 & 0.052 & 1.11 & 14.0 & 0.52 & 7.8 & 190 & 2.93 & 188.16 & 6.72 & 78.4 \\
\hline $40-60$ & 37.5 & 20.0 & 42.5 & $\mathrm{C}$ & 4.60 & 0.040 & 0.81 & 11.6 & 0.44 & 8.6 & 1.50 & 1.79 & 188.16 & 4.48 & 56.0 \\
\hline $60-80$ & 34.3 & 25.7 & 40.0 & $\mathrm{C}$ & 4.60 & 0.023 & 0.42 & 10.0 & 0.32 & 9.0 & 1.30 & 1.86 & 125.44 & 2.24 & 50.4 \\
\hline \multicolumn{16}{|c|}{ Profile 13 (Khumti I) } \\
\hline $0-20$ & 38.5 & 20.0 & 41.5 & $\mathrm{C}$ & 4.70 & 0.082 & 1.47 & 16.4 & 0.48 & 6.1 & 4.20 & 4.40 & 313.60 & 17.92 & 218.4 \\
\hline $20-40$ & 36.0 & 22.5 & 41.5 & $\mathrm{C}$ & 4.80 & 0.075 & 1.17 & 15.8 & 0.56 & 6.5 & 3.50 & 4.20 & 250.88 & 6.72 & 162.4 \\
\hline $40-60$ & 33.5 & 22.5 & 44.0 & $\mathrm{C}$ & 5.00 & 0.076 & 0.78 & 15.0 & 0.32 & 8.4 & 2.60 & 4.80 & 188.16 & 4.48 & 154.6 \\
\hline $60-80$ & 30.0 & 20.2 & 49.8 & $\mathrm{C}$ & 5.00 & 0.032 & 0.75 & 14.6 & 0.32 & 9.9 & 1.90 & 2.90 & 188.16 & 8.96 & 112.0 \\
\hline \multicolumn{16}{|c|}{ Profile 14 (Khumti II) } \\
\hline $0-20$ & 28.8 & 28.4 & 42.8 & $\mathrm{C}$ & 4.70 & 0.154 & 1.56 & 17.8 & 0.44 & 6.6 & 4.00 & 3.80 & 313.60 & 15.69 & 196.0 \\
\hline $20-40$ & 34.8 & 22.5 & 42.7 & $\mathrm{C}$ & 4.50 & 0.064 & 1.02 & 16.8 & 0.48 & 7.9 & 1.90 & 2.30 & 250.88 & 13.44 & 151.2 \\
\hline $40-60$ & 38.0 & 18.4 & 43.6 & $\mathrm{C}$ & 4.60 & 0.038 & 0.57 & 13.0 & 0.56 & 12.0 & 1.30 & 2.20 & 188.16 & 13.44 & 168.0 \\
\hline $60-80$ & 26.2 & 26.8 & 47.0 & $\mathrm{C}$ & 4.40 & 0.021 & 0.42 & 14.0 & 0.32 & 10.0 & 1.20 & 2.30 & 125.44 & 8.96 & 140.0 \\
\hline
\end{tabular}




\begin{tabular}{|c|c|c|c|c|c|c|c|c|c|c|c|c|c|c|c|}
\hline $\begin{array}{l}\text { Depth } \\
(\mathrm{cm})\end{array}$ & $\begin{array}{c}\text { Sand } \\
(\%)\end{array}$ & $\begin{array}{l}\text { Silt } \\
(\%)\end{array}$ & $\begin{array}{l}\text { Clay } \\
(\%)\end{array}$ & Texture & $\mathrm{pH}$ & $\begin{array}{l}\mathrm{EC} \\
(\mathrm{dS} \\
\left.\mathrm{m}^{-1}\right)\end{array}$ & $\underset{\left(\mathrm{gkg}^{-1}\right)}{\mathrm{OC}}$ & $\begin{array}{c}\mathrm{CEC} \\
{[\mathrm{cmol}} \\
\left(\mathrm{p}^{+}\right) \\
\left.\mathrm{kg}^{-1}\right]\end{array}$ & $\begin{array}{c}\mathrm{Fe}_{2} \mathrm{O}_{3} \\
(\%)\end{array}$ & $\begin{array}{c}\mathrm{Al}_{2} \mathrm{O}_{3} \\
(\%)\end{array}$ & $\begin{array}{c}\mathrm{Ca} \\
{[\mathrm{cmol}} \\
\left(\mathrm{p}^{+}\right) \\
\left.\mathrm{kg}^{-1}\right]\end{array}$ & $\begin{array}{c}\mathrm{Mg} \\
{[\mathrm{cmol}} \\
\left(\mathrm{p}^{+}\right) \\
\left.\mathrm{kg}^{-1}\right]\end{array}$ & $\begin{array}{c}\mathrm{N} \\
(\mathrm{kg} \\
\left.\mathrm{ha}^{-1}\right)\end{array}$ & $\begin{array}{c}\mathrm{P} \\
(\mathrm{kg} \\
\left.\mathrm{ha}^{-1}\right)\end{array}$ & $\begin{array}{c}\mathrm{K} \\
(\mathrm{kg} \\
\left.\mathrm{ha}^{-1}\right)\end{array}$ \\
\hline \multicolumn{16}{|c|}{ Profile 15 (Khomjaron) } \\
\hline $0-20$ & 24.3 & 19.2 & 56.5 & $\mathrm{C}$ & 4.90 & 0.122 & 3.06 & 16.0 & 0.40 & 10.2 & 2.90 & 2.10 & 313.60 & 17.92 & 224.0 \\
\hline $20-40$ & 19.3 & 22.5 & 58.2 & $\mathrm{C}$ & 4.60 & 0.054 & 1.11 & 13.0 & 0.30 & 13.8 & 0.93 & 0.53 & 250.88 & 13.44 & 145.6 \\
\hline $40-60$ & 16.8 & 27.5 & 55.7 & $\mathrm{C}$ & 4.80 & 0.045 & 0.96 & 12.8 & 0.52 & 12.5 & 0.66 & 0.47 & 250.88 & 11.20 & 123.6 \\
\hline $60-80$ & 16.8 & 26.7 & 56.5 & $\mathrm{C}$ & 4.80 & 0.021 & 0.96 & 9.6 & 0.52 & 13.6 & 0.60 & 0.46 & 188.16 & 8.96 & 134.4 \\
\hline \multicolumn{16}{|c|}{ Profile 16 (Khebuching) } \\
\hline $0-20$ & 34.3 & 25.0 & 40.7 & $\mathrm{C}$ & 4.30 & 0.063 & 2.13 & 15.0 & 0.20 & 8.8 & 1.00 & 0.33 & 250.88 & 17.92 & 168.0 \\
\hline $20-40$ & 41.8 & 15.0 & 43.2 & $\mathrm{C}$ & 4.50 & 0.051 & 1.17 & 13.0 & 0.36 & 9.6 & 0.73 & 0.19 & 250.88 & 15.68 & 84.0 \\
\hline $40-60$ & 41.8 & 12.5 & 45.7 & $\mathrm{C}$ & 4.50 & 0.022 & 0.75 & 12.0 & 0.24 & 11.0 & 0.53 & 0.13 & 188.16 & 13.44 & 78.4 \\
\hline $60-80$ & 29.3 & 25.0 & 45.7 & $\mathrm{C}$ & 4.60 & 0.014 & 0.63 & 11.0 & 0.28 & 10.2 & 0.53 & 0.13 & 125.44 & 8.96 & 67.2 \\
\hline
\end{tabular}

The multiple regression equations presented in the Table 3 indicate a predictability value of 77.4 per cent by all factors taken together in the $1^{\text {st }}$ layer. Significant regression with $\mathrm{EC}(3.566 *)$ and $\mathrm{Fe}(0.022 * *)$ in the $2^{\text {nd }}$ layer, $\mathrm{Fe}(0.012 *)$ in the $3^{\text {rd }}$ layer and silt $\left(0.029^{* *}\right)$ and $\mathrm{Cu}\left(0.451^{*}\right)$ in the $4^{\text {th }}$ layer and their predictability were $71.1,75.0$ and 73.7 percent, respectively (Table 3 ).

\subsection{Copper $(\mathrm{Cu})$}

DTPA-extractable $\mathrm{Cu}$ content in the profiles ranged from trace to $0.8 \mathrm{mg} \mathrm{kg}^{-1}$. In all the sixteen profiles, DTPA-extractable $\mathrm{Cu}$ content in the soil was found in adequate range, being $0.2 \mathrm{mg} \mathrm{kg}^{-1}$ as critical value (Lindsay \& Norvell, 1978). DTPA-extractable $\mathrm{Cu}$ content was higher in the surface soils and decreased gradually in all the profiles. Similar results were also reported by various researchers (Gupta et al., 2003, Verma et al., 2007a, Verma et al., 2007b, Athokpam, et al., 2016). The multiple correlation and regression analyses indicated that 46.7, 69.1 and 80.4 per cent variability may be the combine effect of $\mathrm{Fe}, \mathrm{Mn}, \mathrm{Mg}$ and $\mathrm{Zn}$ content in the profile.

\subsection{Iron $(\mathrm{Fe})$}

DTPA-extractable Fe content in the profiles ranged from 5.8 to $42.1 \mathrm{mg} \mathrm{kg}^{-1}$ and is comparable with those reported by Gupta et al. (2003) and Sharma \& Choudhary (2007) in the soils of Madhya Pradesh and north-west Himalaya (H.P.), respectively. The critical limit of the soils is $4.5 \mathrm{mg} \mathrm{kg}^{-1}$ as given by Lindsay \& Norvell (1978), all the profiles have sufficient amounts of $\mathrm{Fe}$ in the soils. It showed significant regression coefficient with $\mathrm{Zn}$ $\left(15.322^{*}, 29.673^{*}\right.$ and $\left.8.288^{*}\right)$ in the $2^{\text {nd }}, 3^{\text {rd }}$ and $4^{\text {th }}$ layers, respectively indicating dynamic equilibrium in the profiles.
Multiple correlation and regression analyses indicated that 73.0, 70.1 and 55.0 percent variability in the DTPA-extractable $\mathrm{Fe}$ in the profiles was due to the combine effect of $\mathrm{Al}_{2} \mathrm{O}_{3}, \mathrm{Cu}, \mathrm{Zn}, \mathrm{Mn}$, $\mathrm{Mg}$ and silt in the soils.

\subsection{Manganese (Mn)}

DTPA-extractable $\mathrm{Mn}$ in the studied profiles varied from 0.35 to $29.55 \mathrm{mg} \mathrm{kg}^{-1}$ with a mean value of $15.58 \mathrm{mg} \mathrm{kg}^{-1}$. The surface soils contained higher $\mathrm{Mn}$ and decreased with increase in the depth (Gupta et al., 2003; Thangasawy et al., 2005; Verma et al., 2007a; Verma et al., 2007b). The studied soil profiles were above the critical limit as suggested by Lindsay \& Norvell (1978), as the critical limit of the soils is $1.0 \mathrm{mg} \mathrm{kg}^{-1}$. Only 4.67 percent of the studied samples were below critical limits. Multiple correlation and regression analyses indicated that 41.1, 65.6 and 64.5 per cent variability of the available $\mathrm{Mn}$ content and could be attributed to the combine effect of $\mathrm{Mg}, \mathrm{Cu}, \mathrm{Fe}, \mathrm{Zn}$ and silt content in the profiles (Table 3).

The variations observed in available micronutrient cations among and within the profiles might be the result of variable intensity of different pedogenic processes taking place during the soil development. The surface layers contained higher amounts of available $\mathrm{Zn}, \mathrm{Cu}, \mathrm{Fe}$ and $\mathrm{Mn}$ which progressively declined with depth in majority of the soil profiles (Table 2). Similar distribution pattern of micronutrient cations within the profiles was also reported by Sharma et al. (1999) and Sharma \& Choudhary (2007). This may be ascribed to low $\mathrm{pH}$ values and higher amounts of organic carbon content in the surface soils. Decomposition of organic matter releases micronutrient cations and some organic acids which in turn help in increasing solubility of micronutrient cations from the soil mineral. Significant positive 
Table 2 DTPA-extractable micronutrient cations $\left(\mathrm{mg} \mathrm{kg}^{-1}\right)$ in the soils

\begin{tabular}{|c|c|c|c|c|c|c|c|c|c|}
\hline Depth $(\mathrm{cm})$ & $\mathbf{Z n}$ & $\mathbf{C u}$ & $\mathrm{Fe}$ & Mn & Depth (cm) & $\mathbf{Z n}$ & $\mathbf{C u}$ & $\mathrm{Fe}$ & Mn \\
\hline \multicolumn{5}{|c|}{ Profile 1 (Longmai Noney II) } & \multicolumn{5}{|c|}{ Profile 2 (Longmai Noney II) } \\
\hline $0-20$ & 0.65 & 0.55 & 31.5 & 10.0 & $0-20$ & 0.45 & 0.45 & 38.2 & 26.6 \\
\hline $20-40$ & 0.20 & 0.25 & 22.3 & 7.0 & $20-40$ & 0.00 & 0.35 & 24.3 & 12.0 \\
\hline $40-60$ & 0.20 & 0.30 & 15.0 & 8.7 & $40-60$ & 0.00 & 0.20 & 22.5 & 10.0 \\
\hline $60-80$ & 0.40 & 0.25 & 13.8 & 9.3 & $60-80$ & 0.10 & 0.20 & 21.5 & 10.1 \\
\hline \multicolumn{5}{|c|}{ Profile 3 (Longmai Noney I) } & \multicolumn{5}{|c|}{ Profile 4 (Longmai Noney I) } \\
\hline $0-20$ & 0.40 & 0.30 & 24.6 & 17.0 & $0-20$ & 0.20 & 0.30 & 27.6 & 18.8 \\
\hline $20-40$ & 0.00 & 0.25 & 22.4 & 11.9 & $20-40$ & 0.40 & 0.20 & 18.7 & 6.9 \\
\hline $40-60$ & 0.10 & 0.15 & 19.6 & 7.1 & $40-60$ & 0.25 & 0.05 & 14.8 & 4.1 \\
\hline $60-80$ & 0.00 & 0.15 & 18.4 & 8.3 & $60-80$ & 0.15 & 0.05 & 14.0 & 5.8 \\
\hline \multicolumn{5}{|c|}{ Profile 5 (Tupul I) } & \multicolumn{5}{|c|}{ Profile 6 (Tupul II) } \\
\hline $0-20$ & 0.70 & 0.20 & 24.4 & 26.1 & $0-20$ & 0.95 & 0.40 & 36.0 & 37.0 \\
\hline $20-40$ & 0.40 & 0.25 & 18.3 & 16.7 & $20-40$ & 0.35 & 0.40 & 32.2 & 33.1 \\
\hline $40-60$ & 0.25 & 0.25 & 16.7 & 12.2 & $40-60$ & 0.40 & 0.40 & 24.2 & 20.7 \\
\hline $60-80$ & 0.45 & 0.20 & 11.5 & 8.3 & $60-80$ & 0.35 & 0.30 & 20.6 & 17.0 \\
\hline \multicolumn{5}{|c|}{ Profile 7 (Nungtek) } & \multicolumn{5}{|c|}{ Profile 8 (Thangal) } \\
\hline $0-20$ & 1.10 & 0.40 & 31.2 & 25.3 & $0-20$ & 1.75 & 0.50 & 45.2 & 21.7 \\
\hline $20-40$ & 0.60 & 0.60 & 33.7 & 22.3 & $20-40$ & 1.20 & 0.40 & 48.5 & 16.5 \\
\hline $40-60$ & 0.50 & 0.55 & 36.7 & 28.8 & $40-60$ & 0.85 & 0.45 & 48.2 & 13.7 \\
\hline $60-80$ & 0.45 & 0.45 & 32.2 & 24.5 & $60-80$ & 0.65 & 0.55 & 40.8 & 7.5 \\
\hline \multicolumn{5}{|c|}{ Profile 9 (Awang Khul) } & \multicolumn{5}{|c|}{ Profile 10 (Rengpang) } \\
\hline $0-20$ & 0.80 & 0.35 & 37.0 & 25.7 & 0.25 & 0.25 & 27.8 & 23.5 & 0.25 \\
\hline $20-40$ & 0.65 & 0.20 & 22.3 & 13.1 & 0.10 & 0.15 & 17.2 & 10.8 & 0.10 \\
\hline $40-60$ & 0.10 & 0.20 & 20.7 & 8.4 & 0.00 & 0.05 & 13.7 & 4.5 & 0.00 \\
\hline $60-80$ & 0.30 & 0.15 & 14.2 & 7.8 & 0.10 & 0.00 & 14.0 & 3.8 & 0.10 \\
\hline \multicolumn{5}{|c|}{ Profile 11 (Longmai I) } & \multicolumn{5}{|c|}{ Profile 12 (Longmai II) } \\
\hline $0-20$ & 0.70 & 0.55 & 22.0 & 40.0 & $0-20$ & 0.70 & 0.30 & 42.1 & 24.0 \\
\hline $20-40$ & 0.30 & 0.65 & 21.2 & 33.3 & $20-40$ & 0.50 & 0.40 & 36.6 & 15.6 \\
\hline $40-60$ & 0.25 & 0.65 & 13.6 & 20.9 & $40-60$ & 0.10 & 0.45 & 23.0 & 8.8 \\
\hline $60-80$ & 0.35 & 0.80 & 12.4 & 27.9 & $60-80$ & 0.25 & 0.25 & 18.2 & 6.8 \\
\hline \multicolumn{5}{|c|}{ Profile 13 (Khumti I) } & \multicolumn{5}{|c|}{ Profile 14 (Khumti II) } \\
\hline $0-20$ & 0.65 & 0.25 & 31.8 & 34.7 & $0-20$ & 0.65 & 0.20 & 25.6 & 30.9 \\
\hline $20-40$ & 0.30 & 0.25 & 28.7 & 28.8 & $20-40$ & 0.25 & 0.20 & 20.6 & 25.4 \\
\hline $40-60$ & 0.20 & 0.25 & 21.0 & 18.4 & $40-60$ & 0.20 & 0.25 & 16.4 & 20.1 \\
\hline $60-80$ & 0.00 & 0.15 & 13.8 & 9.35 & $60-80$ & 0.15 & 0.20 & 11.5 & 19.0 \\
\hline \multicolumn{5}{|c|}{ Profile 15 (Khomjaron } & \multicolumn{5}{|c|}{ Profile 16 (Khebuching) } \\
\hline $0-20$ & 0.60 & 0.40 & 21.7 & 24.4 & $0-20$ & 0.60 & 0.10 & 36.3 & 2.1 \\
\hline $20-40$ & 0.10 & 0.25 & 10.2 & 2.1 & $20-40$ & 0.10 & 0.00 & 18.1 & 0.8 \\
\hline $40-60$ & 0.15 & 0.00 & 7.4 & 1.1 & $40-60$ & 0.10 & 0.00 & 10.0 & 0.5 \\
\hline $60-80$ & 0.20 & 0.05 & 7.5 & 1.4 & $60-80$ & 0.15 & 0.00 & 5.8 & 0.4 \\
\hline
\end{tabular}

Journal of Experimental Biology and Agriculture Science http://www.jebas.org 
Table 3 Effect of soil characteristics on predictability of micronutrient cations

\begin{tabular}{|c|c|c|}
\hline Micronutrients & Equations & $R^{2} \times 100$ \\
\hline \multicolumn{3}{|c|}{ Available Zn } \\
\hline $\begin{array}{l}1^{\text {st }} \text { layer }(0-20 \mathrm{~cm}) \\
2^{\text {nd }} \text { layer }(20-40 \mathrm{~cm}) \\
3^{\text {rd }} \text { layer }(40-60 \mathrm{~cm}) \\
4^{\text {th }} \text { layer }(60-80 \mathrm{~cm})\end{array}$ & $\begin{array}{l}-0.523+3.868^{*} \mathrm{EC}+0.024^{* *} \mathrm{Fe}+0.002 \mathrm{Ca} \\
-0.407+3.566^{*} \mathrm{EC}+0.022^{* *} \mathrm{Fe}-0.068 \mathrm{Ca}+0.001 \mathrm{~K}-0.003 \mathrm{Mg} \\
-0.496+0.002 \mathrm{~N}+0.137 \mathrm{Cu}+0.012^{*} \mathrm{Fe}+0.00 \mathrm{Mn} \\
0.832-0.005 \mathrm{Mg}-0.029^{* *} \mathrm{Silt}+0.451^{*} \mathrm{Cu}-0.001 \mathrm{Fe}\end{array}$ & $\begin{array}{l}\text { 77.4*** } \\
\text { 71.1** } \\
\text { 75.0** } \\
\text { 73.7** }\end{array}$ \\
\hline \multicolumn{3}{|c|}{ Available $\mathrm{Cu}$} \\
\hline $\begin{array}{l}2^{\text {nd }} \text { layer }(20-40 \mathrm{~cm}) \\
3^{\text {rd }} \text { layer }(40-60 \mathrm{~cm}) \\
4^{\text {th }} \text { layer }(60-80 \mathrm{~cm})\end{array}$ & $\begin{array}{l}0.044+0.005 \mathrm{Fe}+0.008^{*} \mathrm{Mn} \\
0.070+0.002 \text { clay }+0.003 \mathrm{Fe}+0.031 \mathrm{Zn}+0.014^{*} \mathrm{Mn}+0.01 \mathrm{Mg} \\
-0.115+0.003 \mathrm{Fe}+0.344 \mathrm{Zn}+0.016^{* *} \mathrm{Mn}+0.033 \mathrm{Mg}\end{array}$ & $\begin{array}{l}46.7^{*} \\
69.1^{* *} \\
80.4^{* *}\end{array}$ \\
\hline \multicolumn{3}{|c|}{ Available Fe } \\
\hline $\begin{array}{l}2^{\text {nd }} \text { layer }(20-40 \mathrm{~cm}) \\
3^{\text {rd }} \text { layer }(40-60 \mathrm{~cm}) \\
4^{\text {th }} \text { layer }(60-80 \mathrm{~cm})\end{array}$ & $\begin{array}{l}26.322-1.369 \mathrm{Al}_{2} \mathrm{O}_{3}+15.518 \mathrm{Cu}+15.322 * \mathrm{Zn}+0.240 \mathrm{Mg} \\
21.929-1.171 \mathrm{Al}_{2} \mathrm{O}_{3}+3.552 \mathrm{Cu}+29.673 * * \mathrm{Zn}+0.119 \mathrm{Mn} \\
37.159-0.335 \mathrm{Silt}+8.288^{*} \mathrm{Zn}-1.929 \mathrm{Al}_{2} \mathrm{O}_{3}+11.156 \mathrm{Cu}\end{array}$ & $\begin{array}{l}\mathbf{7 3 . 0} * * \\
\mathbf{7 0 . 7} * * \\
\mathbf{5 5 . 0} *\end{array}$ \\
\hline \multicolumn{3}{|c|}{ Available Mn } \\
\hline $\begin{array}{l}2^{\text {nd }} \text { layer }(20-40 \mathrm{~cm}) \\
3^{\text {rd }} \text { layer }(40-60 \mathrm{~cm}) \\
4^{\text {th }} \text { layer }(60-80 \mathrm{~cm})\end{array}$ & $\begin{array}{l}2.794+36.548 * \mathrm{Cu}+0.639 \mathrm{Mg} \\
1.877+27.805 * \mathrm{Cu}+0.059 \mathrm{Fe}+0.601 \mathrm{Zn}+0.768 \mathrm{Mg} \\
7.442+0.465 \mathrm{Silt}+30.154 * \mathrm{Cu}\end{array}$ & $\begin{array}{l}\text { 41.1* } \\
\text { 65.6** } \\
64.5^{* *}\end{array}$ \\
\hline
\end{tabular}

regression coefficients of EC with DTPA-extractable micronutrients have also been reported by Randhawa \& Singh (1995) and Sharma et al. (2006).

\section{Conclusion}

The surface horizons of the soil profiles were fairly high in organic carbon and adequate in all the macro and micronutrients content. However, in the sub-surface horizons especially $40 \mathrm{~cm}$ to $80 \mathrm{~cm}$ depth, the content of all the nutrients decreased below critical limits for the proper growth of the fruit trees. Thus, for the deep rooted fruit crops requires a well balanced nutrition of both macro and micronutrients, especially $\mathrm{Zn}$ to the root zones for proper growth and development.

\section{Conflict of interest}

Authors would hereby like to declare that there is no conflict of interests that could possibly arise.

\section{References}

Athokpam HS, Zimik VS, Chongtham N, Devi KN, Singh NB, Watham L, Sharma PT, Athokpam H (2016) Profile distribution of micronutrient cations in citrus orchard of Ukhrul district, Manipur (India). International Journal of Agriculture, Environment and Biotechnology $9: 691-697$.
Borah DK, Bordoloi PK, Karmakar RM, Baruah NG, Das M (1987) Practical manual for fundamental of soil science (Part-III), Jorhat, Assam.

Chopra SL, Kanwar JS (1976) Analytical Agricultural Chemistry. Kalyani Publishers, Ludhiana-New Delhi.

Gupta N, Trivedi SK, Bansali KN, Kaul RK (2003) Vertical distribution of micronutrient cations in some soil series of north Madhya Pradesh. Journal of Indian Society of Soil Science 51:517-522.

Jackson ML (1973) Soil chemical analysis. Prentice-Hall of India Private Limited, New Delhi.

Khanday MUDD, Ram D, Wani JA, Ali T (2017) Vertical distribution of nutrients of the soils of Namblan sub-catchment of Jhelum basin of Srinagar district in Khashmir valley. International Journal of Current Microbiology and Applied Sciences $6: 375-381$.

Kumar CJN, Rajangam J, Balakrishnan K, Bora L (2017) Influence of foliar fertilization of micronutrients on leaf micro nutrient status of mandarin orange (Citrus reticulate Blanco.) in lower Pulney hills. International Journal of Current Microbiology and Applied Sciences $6: 516522$. 
Lindsay WL, Norvell WA (1978) Development of DTPA soil test for $\mathrm{Zn}, \mathrm{Fe}, \mathrm{Mn}$ and $\mathrm{Cu}$. Soil Science Society of American Journal 42:421-428.

Panse VG, Sukhatme PV (1961) Statistical Methods for Agricultural Workers, ICAR, New Delhi.

Ram RA, Bose (2000) Effect of foliar application of magnesium and micronutrients on growth, yield and fruit quality of mandarin orange (Citrus reticulate Blanco). Indian Journal of Horticulture 57: 215-220.

Randhawa HS, Singh P (1995) Distribution of zinc fractions in alluvium derived soils of Punjab. Journal of the Indian Society of Soil Science 43:124-126.

Rattan RK, Patel KP, Manjaiah KM, Datta SP (2009) Micronutrients in soil, plant, animal and human health. Journal of the Indian Society of Soil Science 57:546-558.

Sen TK, Dubey PN, Maji AK, Chamuah GS (1997) Status of micronutrients in some dominant soils of Manipur. Journal of the Indian Society of Soil Science 45:388-390.

Sharma BD, Jassal HS, Swahney JS, Sidhu PS (1999) Micronutrient distribution in different physiographic unit of Siwalik hills of semiarid tract of Punjab. Arid Soil Research and Rehabilitation 13:189-200.

Sharma JC, Choudhary SK (2007) Vertical distribution of micronutrient cations in relation to soil characteristics in lower Shiwaliks of Solan district in North-west Himalayas. Journal of the Indian Society of Soil Science 55:40-44.

Sharma VK, Sanjai K, Dwivedi, Tripathi D, Ahmed Z (2006) status of available major and micronutrients in the soil of different blocks of Leh district of cold arid region of Ladakh in relation to soil characteristics. Journal of the Indian Society of Soil Science $54: 248-250$.

Singh KMS, Dhankar SS (1989) Influence of soil characteristics on profile distribution of DTPA- extractable micronutrient cations. Indian Journal of Agricultural Sciences 59 : 331-334.

Soni U, Thakre B, Verma O (2017) Effect of micronutrients on growth, vigour and fruit weight of Nagpur mandarin (Citrus reticulate Blanco.) in Satpura plateau region, India. International Journal of Current microbiology and Applied Sciences 6:435-440.

Srivastava PP, Pandiaraj T, Das S, Sinha AK (2017) Assessment of micronutrient status of soil under Tasar host plant growing regions in Jashpur district, Chhattisgarh State. Imperial Journal of Interdisciplinary Research $3: 1080-1083$.

Takkar PN, Mann MS (1975) Evaluation of analytical methods of estimation of available zinc and response of applied zinc in major soil series of Ludhiana, Punjab. Agrochemicals 19:420-430.

Tariq M, Sharif M, Shah Z, Khan R (2007) Effect of foliar application of micronutrients on the yield and quality of sweet orange (Citrus cinensis L.). Pakistan Journal of Biological Sciences $10: 1823-1828$.

Thangasawy A, Naidu MVS, Ramavatharam N, Reddy CL (2005) Characterization, classification and evaluation of soil resources in Sivagiri micro-watershed of Chittoor district in Andhra Pradesh for sustainable land used planning. Journal of the Indian Society of Soil Science 53:515-520.

Verma VK, Setia RK, Sharma PK (2007b) Distribution of micronutrient cations in different physiographic units of semi-arid region of Punjab. Agropedology 18: 58-65.

Verma VK, Setia RK, Sharma PK, Khurana MPS, Kang GS (2007a) Pedopheric distribution of micronutrient cations in soil developed on various landforms in north-east Punjab. Journal of the Indian Society of Soil Science 55:515-520.

Vijaya HM, Godara RK, Singh S, Sharma N (2017) Effect of exogenous application of micronutrients on growth and yield of Kinnow mandarin under semi-arid zone of Haryana. Journal of Pharmacognosy and Phytochemistry $6: 733: 735$. 